\title{
Some Aspects of Coherent Epitaxial Deposits
}

\author{
Pierre Müller and Raymond Kern \\ Centre de Recherche sur les Mécanismes de la Croissance Cristalline $\left({ }^{*}\right)$, Campus de Luminy, \\ case 913, 13288 Marseille Cedex 9, France
}

(Received: July 03; Accepted: October 10, 1997)

PACS.68.10.Cr - Surface energy (surface tension, interface tension, angle of contact, etc.)
PACS.68.10.Gw - Interface activity, spreading
PACS.68.55.-a - Thin film structure and morphology

\begin{abstract}
When wetting is complete, coherent epitaxies on a thick planar substrate B build up $z$ continuous pseudomorphous layers $\mathrm{A}$ which are fully strained according to their natural misfit $m$. The so-accumulated strain energy density is responsible for some remarkable facts: 1) Each layer is formed at precise undersaturation. At saturation there is a specific number of wetting layers $z_{0}$. 2) Such layers accumulate strain energy and then may relax plastically by interfacial dislocations at a critical thickness $z_{\mathrm{d} 1}$. 3) At some other critical thickness $z_{\mathrm{SK}}$, $\left(z_{0}<z_{\mathrm{SK}}<z_{\mathrm{d} 1}\right)$, the monolayer growth becomes less stable than layer thickening, what leads to three-dimensional (3D) islanding on the $z_{\text {sk }}$ wetting layers, called Stranski-Krastanov (SK) growth. Such islands laterally relax and, since coherent with their substrate, drag the substrate which relaxes too. 4) If the equilibrium shape ratio $r$ of non misfitted 3D deposit $(m=0)$ depends on the wetting, for a misfitted $(m \neq 0)$ and relaxed crystal, the strain opposes to wetting, so that the higher the elastic energy, the greater the shape ratio $r_{m}$. 5) Since the equilibrium shape of a $3 \mathrm{D}$ crystal depends upon strain, at each dislocation entrance the shape ratio of the crystal varies in a sudden way.
\end{abstract}

\section{Introduction}

The practical importance of semiconductors systems leads to a renewal of the studies of coherent epitaxial growth on mismatched substrate. The aim of this paper is to illustrate how the elastic energy stored in such strained systems leads to new specific phenomena. For this we will do a synthesis of some published results about crystal growth and add our original contribution about the description of the elastic relaxation and its implications on the equilibrium shape of a deposited crystal. For some reviews on epitaxial growth, one should refer to [1-5]. For the sake of simplicity in the following we will consider a crystal A grown on a semi-infinite crystal $\mathrm{B}$, both being cubic with parameter $a$ and $b$ and do not mix. A and B differ by a natural misfit $m=(b-a) / a$. All surface stresses effect will be neglected.

$\left(^{*}\right)$ Associé aux Universités Aix-Marseille II et III. 


\section{Crystal Growth Mode on a Mismatched Substrate}

\subsection{D and 3D Growth without Elastic Relaxation}

We consider a thermodynamic process where a free slice of crystal A is formed from a vapor, then stretched to be accomodated on the substrate B. Neglecting the surface stress effects the free energy change during the process writes as the sum of 4 terms respectively due to the bulk chemical energy (connected to the chemical potential change $\Delta \mu$ per molecule), to the formation of the surfaces of $\mathrm{A}$ (connected to the surface energy $\sigma_{\mathrm{A}}$ of $\mathrm{A}$ ), to the elastic deformation of the crystal (involving the natural misfit $m$, the Young' modulus $E_{\mathrm{A}}$ and the Poisson's ratio $\nu_{\mathrm{A}}$ of $\mathrm{A}$ ) and the adhesion energy density $\beta_{\mathrm{AB}}$. When depositing $z$ monolayers of $\mathrm{A}$, the free energy change, per unit area of $\mathrm{B}$ (having $n_{s}$ sites) writes:

$$
\Delta f(z)=-\Delta \mu n_{s} z+\varepsilon_{\mathrm{A}} m^{2} n_{s} z+\Phi_{\mathrm{AB}}(z)
$$

where $\Delta \mu$ is called supersaturation when positive, $\varepsilon_{\mathrm{A}} m^{2}=E_{\mathrm{A}} v m^{2} /\left(1-\nu_{\mathrm{A}}\right)$ is the stored elastic energy per atom ( $v$ the atomic volume) and $\Phi_{\mathrm{AB}}(z)$ the unwetting energy where intervenes a size effect $f(z)$. Indeed since manipulating monolayers the usual macroscopic wetting energy $\Phi_{\mathrm{AB}}=\left(2 \sigma_{\mathrm{A}}-\beta_{\mathrm{AB}}\right)$ of semi infinite crystal has to be discretisized. A convenient and meaningful way $\left({ }^{1}\right)$ to proceed $[6]$ is to take the form $\Phi_{\mathrm{AB}}(z)=\Phi_{\mathrm{AB}}\left(1-\mathrm{e}^{z / \zeta}\right)$, where $\zeta$ is a screening factor. In the case of incomplete wetting, $\Phi_{\mathrm{AB}}>0$, the free energy (1) only decreases for $\Delta \mu>\varepsilon_{\mathrm{A}} m^{2}$ provided a critical number of layers $z_{m}$ is overpassed. However since the thick layers may be formed at the lowest supersaturation such a system is completely unstable. In fact 3D islands may form, at $\Delta \mu>\varepsilon_{\mathrm{A}} m^{2}$. It is easily shown that their equilibrium shape ratio $r_{0}$ is

$$
r_{0}=\frac{h}{\ell}=\frac{\Phi_{\mathrm{AB}}}{2 \sigma_{\mathrm{A}}}=1-\frac{\beta_{\mathrm{AB}}}{2 \sigma_{\mathrm{A}}}
$$

that means is size independent (Wulf-Kaishew's theorem $[7,8]$, the shape being a square $\ell^{2}$ base rhombus of height $h$ ). The higher the adhesion energy (in respect to twice the surface energy of the deposit), the flatter the equilibrium shape.

In the opposite case $\Phi_{\mathrm{AB}}<0$ (perfect wetting) from (1) it is seen that for $\Delta \mu<\varepsilon_{\mathrm{A}} m^{2}$ that means even at undersaturation $\Delta \mu<0<\varepsilon_{\mathrm{A}} m^{2}$, the free energy decreases up to a minimum. To each integer $z$ corresponds a given supersaturation $\Delta \mu_{z}$, where takes place a first order transition: a layer is formed. Putting the discrete derivative of (1) zero brings $\Delta \mu_{z}=\varepsilon_{\mathrm{A}} m^{2} n_{\mathrm{s}}-\left|\Phi_{\mathrm{AB}}\right|\left(1-\mathrm{e}^{-1 / \zeta}\right) \mathrm{e}^{-z / \zeta}$. Up to saturation $\Delta \mu=0$ there builds up a finite number of layers $z_{0}$ given by

$$
\mathrm{e}^{-z_{0}}=\varepsilon_{\mathrm{A}} m^{2} n_{\mathrm{s}} /\left|\Phi_{\mathrm{AB}}\right|\left(1-\mathrm{e}^{-1 / \zeta}\right)
$$

depending upon the strain over wetting energy ratio. This result is quite more general. In the case of van der Waals systems it was found and experimentally confirmed (see [3]) the functional $z_{0}^{-3}$ dependency. These layers grow at $\Delta \mu<0$ that means reversibly. For $\Delta \mu>0$, that means at supersaturation, reversibility is no more found, but $3 \mathrm{D}$ islanding is observed. We will clear this up in $1.2\left({ }^{2}\right)$.

Let us note that during its thickening a film accumulates strain energy $\varepsilon_{\mathrm{A}} m^{2} n_{\mathrm{s}} z$ which may become prohibitive so that the growing layers may become unstable by the introduction of

\footnotetext{
$\left({ }^{1}\right)$ For the surface melting phenomena similar dependencies are used.

$\left({ }^{2}\right)$ Let us mention that electrochemical deposition is well suited for studying layer formation, it is then called underpotential deposition.
} 
some plastic strain defects. Interfacial explicit dislocations are the most usual and well studied strain releaser $[1,2,5]$. The prerequisit of dislocation introduction is the reduction of the total elastic energy or the reduction of the misfit $m$ to $m^{\prime}=\left(a^{\prime}-b^{\prime}\right) / a^{\prime}<m$. The number of dislocations per unit length is $\left(m-m^{\prime}\right) / a^{\prime}$ and the self energy per unit area of interface, of two orthogonal arrays of dislocations roughly is $2 \frac{m-m^{\prime}}{a^{\prime}} \frac{b^{2} E_{\mathrm{AB}}}{2 \pi} \ln (1+z)$ [1], (b Burgers vector modulus in the glide plane). Minimizing the sum of this energy and the elastic energy of the film $\varepsilon_{\mathrm{A}} n_{\mathrm{s}}\left(m^{\prime}\right)^{2} z$ (having now a reduced misfit $m^{\prime}$ ) there is an equilibrium misfit $m_{\text {eq }}^{\prime}$ provided the total minimized energy is smaller than $\varepsilon_{\mathrm{A}} n_{\mathrm{s}} m^{2} z$ what can be verified. There is roughly $m_{\mathrm{eq}}^{\prime} \approx \frac{K}{4 \pi(1+K)} \frac{\ln (1+z)}{z}$ with $K=E_{\mathrm{B}} / E_{\mathrm{A}}, a \approx a^{\prime} \approx b \approx b^{\prime}$ [1]. Such a reversible introduction of dislocations therefore reduces the mean strain in the layers which decreases as the inverse (roughly) of the number of deposited layers $z$. The relative stiffness of substrate to deposit plays moderately since we took as interfacial modulus $E_{\mathrm{AB}}$ the harmonic mean $1 / E_{\mathrm{AB}}=1 / E_{\mathrm{A}}+1 / E_{\mathrm{B}}$. Starting dislocation free growth for a specific system, say $m_{\text {eq }}^{\prime}=m$, the strain stays constant but at thickness $z_{\mathrm{d} 1}$ the dislocations may introduce and strain decay starts. $z_{\mathrm{d} 1}$ is called the critical thickness of this film.

\section{The Apparent Paradox of the Stranski-Krastanov Growth Mode}

For non dislocated layers $z_{0}<z<z_{\mathrm{d} 1}$ at $\Delta \mu>0$, a growth change called Stranski- Krastanov growth (SK) may appear: upon the $2 \mathrm{D}$ layers of some critical thickness $z=z_{\mathrm{SK}}, 3 \mathrm{D}$ islanding of material A is preferred [9]. Such a well experimentally established behavior is astonishing. Indeed if $\Phi_{\mathrm{AB}}<0$ (necessary for the 2D growth of the underlying layers), the size dependent wetting energy of a crystal A (height $h$ ) sitting on $z$ underlying layers of A deposited on $\mathrm{B}$ writes $\Phi_{\mathrm{A} 2 \mathrm{D}, \mathrm{A} 3 \mathrm{D}}=-\left|\Phi_{\mathrm{AB}}\right|\left(1-\mathrm{e}^{-z / \zeta}\right) \sum_{n=1}^{n=h} \mathrm{e}^{-(n-1) / \zeta}[6]$ which of course is a negative quantity. In such a case the 3D material A should spread over the SK layers A. As every paradox it is only an apparent one. In the past (see for review $[3,4]$ ) it was argued that the SK layers A have very different $2 \mathrm{D}$ structures in respect to that one of $3 \mathrm{D}$ crystals so that there may be intrisically $\Phi_{\mathrm{A} 2 \mathrm{D}, \mathrm{A} 3 \mathrm{D}}>0$. Cases of exotic $2 \mathrm{D}$ structures have been found but SK growth exists also for $2 \mathrm{D}$ layers of $\mathrm{A}$ having the same structure as a parallel plane in the $3 \mathrm{D}$ crystal of $\mathrm{A}$. Therefore a more general explanation has to be found.

The weak point of the "paradox" is that the 2D layers and 3D crystal even of same structure, are in different state of strain. In other terms the mean strain energy per atom differs in the layer and in the island. The strain in the layer $\mathrm{A}$ is described by $\varepsilon_{x x}^{\mathrm{A}}=\varepsilon_{y y}^{\mathrm{A}}=-(1-$ $\left.\nu_{\mathrm{A}}\right) \varepsilon_{z z}^{\mathrm{A}} / 2 \nu_{\mathrm{A}} \cong m=(b-a) / a$. The corresponding stresses are given by Hookes law with the condition that the layer free surface must have zero normal stress $\left(^{3}\right) \sigma_{z z}^{\mathrm{A}}=0$. For the $3 \mathrm{D}$ crystal furthermore, the lateral faces being free, they must have zero normal stress, respectively $\sigma_{x x}^{\mathrm{A}}, \sigma_{y y}^{\mathrm{A}}$ along the four surfaces of areas $h \ell$. Clearly this additional condition changes the state of strain and stress inside the island in respect to the layer. It is said that the island relaxes elastically from its non relaxed state $m$ to an unhomogeneous state $\varepsilon_{\alpha \beta}^{\mathrm{A}}(x, y, z)$ which has to be evaluated. This elastic relaxation is the physical origin of the coherent SK growth mode as we will show in the next paragraph.

\footnotetext{
$\left({ }^{3}\right)$ No confusion is possible with surface energy notation $\sigma_{\mathrm{A}}$ of $\mathrm{A}$, the mechanical stresses of $\mathrm{A}$ being written $\sigma_{\alpha \beta}^{\mathrm{A}}$ with $\alpha, \beta=x, y, z$ the coordinates, $x, y$ in the basal surface of $\mathrm{A}, z$ normal.
} 

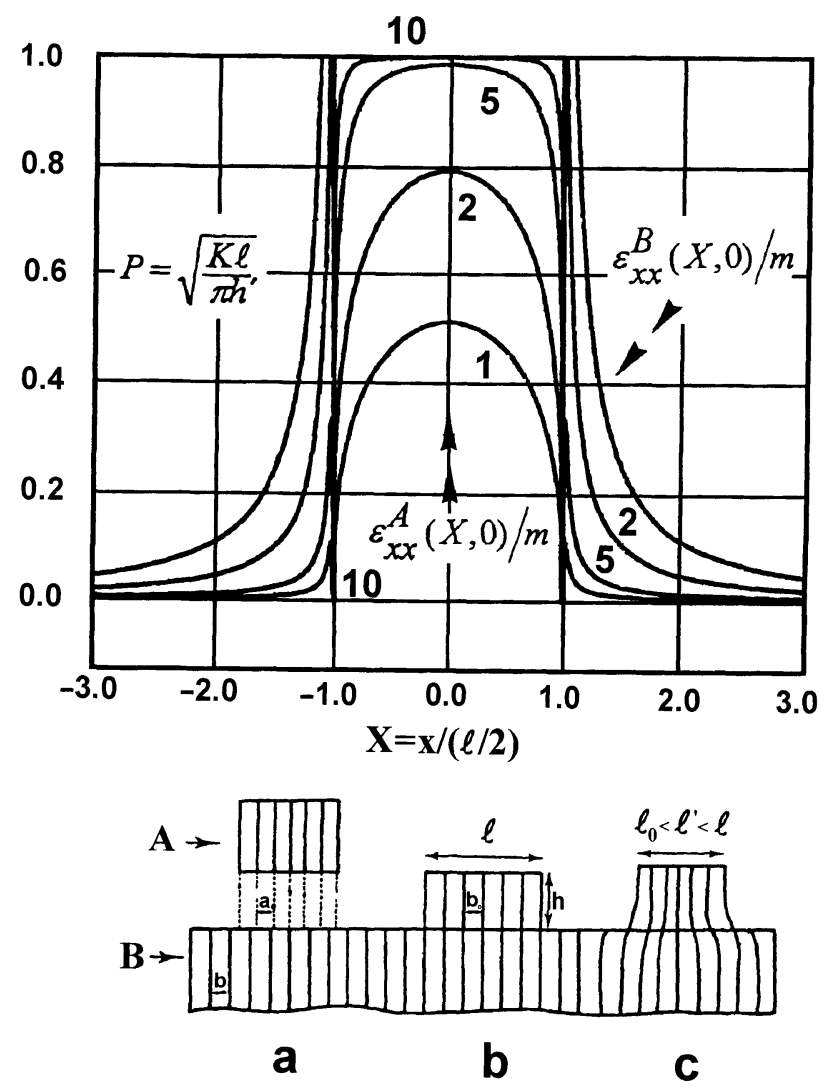

Fig. 1. - Lateral misfit normalized strain field inside $-1<X<1$ and outside $|X|>1$ the contact area of a deposit crystal A. Curves parametrized in $P=10,5,2,1$ according to the size $\ell$ and height $h$ of the deposit A and the relative stiffness $K$ of the substrate B $\left(h^{\prime}=h\langle f\rangle\right)$. In the lower part there is a sketch of the relaxation process.

\section{Description of the Elastic Relaxation}

We will suppose that the epitaxial contact between a 3D deposited crystal and its lattice mismatched substrate $B$ remains coherent during the elastic relaxation of the crystal. In this case, during the relaxation the substrate $\mathrm{B}$ is perfectly dragged by the deposit. In the interface $\mathrm{d} \varepsilon_{x x}^{\mathrm{B}}=\mathrm{d} \varepsilon_{x x}^{\mathrm{A}}$ or $\varepsilon_{x x}^{\mathrm{A}}=\varepsilon_{x x}^{\mathrm{B}}+m$ so that there is continuity of the lattices across the interface. Any relaxation of the deposit $\left|\varepsilon_{x x}^{\mathrm{A}}\right|<|m|$ produces a substrate deformation. This deformation has been calculated by assuming that the epitaxial contact can be described by a distribution of elastic monopoles along the periphery of the island [11] or by a distribution of force dipoles in the contact area [12]. A self consistent treatment is however necessary [13] to solve the stressstrain relaxation. That means the force distribution along the contact area has to change when the substrate deformation yields. This can only be done easily for a plane strain system [13] but can be safely extended to the square base $h \times \ell \times \ell$ island [14]. The lower part of Figure 1 gives a saggital section sketching the process of deformation and then the relaxation of the crystal dragging the substrate. Figure 1 also gives the quantitative result of the lateral strain $\varepsilon_{x x}$ along the interface $z=0$, as a function of the reduced variable $X=x /(\ell / 2)$ for curves 


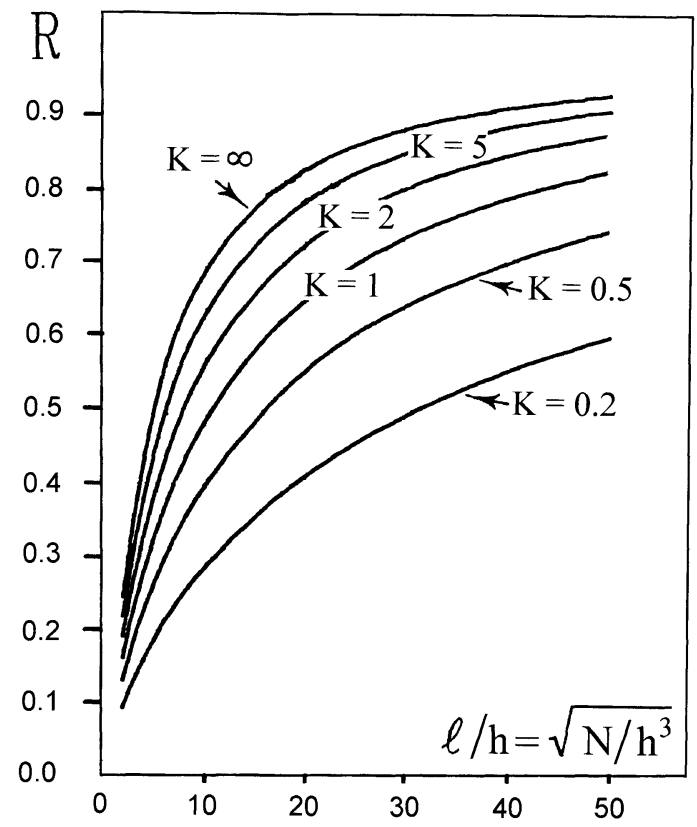

Fig. 2. - Elastic energy relaxation factor $R$ as a function of the shape factor $h / \ell$ of the deposit, parametrized in the relative stiffness $K$ of substrate /deposit.

parametrized by $P=\sqrt{K \ell / \pi h\langle f\rangle}$. In this factor $P$ one recognizes the shape ratio $r=h / \ell$, the relative stiffness $K=E_{\mathrm{B}} / E_{\mathrm{A}}$ (to be put $K=1$ for the SK case), $\langle f\rangle$ is a factor describing how the $3 \mathrm{D}$ crystal relaxes with height $h$ so that $h\langle f\rangle<h$ then is an effective thickness. In Figure 1 it is seen that the reduced strain $\varepsilon_{x x}^{\mathrm{A}} / m$ at the bottom of the island is everywhere smaller than unity (strain relaxation). It reaches a maximum value in the middle and vanishes at the borders. Outside the contact area the substrate shows a deformation $\varepsilon_{x x}^{\mathrm{B}} / m$ falling down rapidly. The stiffer the substrate, the smaller this deformation. The analytical dependencies are

$$
\begin{aligned}
-1<X<1, \quad \frac{\varepsilon_{x x}^{\mathrm{A}}(X, 0)}{m} & =M(X)=1+\mathrm{e}^{-P \sqrt{2}}-\mathrm{e}^{-P \sqrt{|1+X|}}-\mathrm{e}^{-P \sqrt{|1-X|}} \\
|X|>1, \quad \frac{\varepsilon_{x x}^{\mathrm{B}}(X, 0)}{m} & =\left(\frac{2}{\pi P}\right)^{2}\left[X^{2}-1\right]^{-1} .
\end{aligned}
$$

From these data the minimal energy stored in that system can be evaluated. It writes

$$
W_{\min }=\varepsilon_{\mathrm{A}} h \ell^{2} m^{2} R(h / \ell)
$$

so that the non relaxed energy of the island is multiplied by a factor $R(h / \ell)<1$, we call relaxation factor. $R(h / \ell)$ only depends upon the shape ratio $r=h / \ell$ and the relative stiffness $K$. In Figure 2 we give the $R$ over $1 / r$ dependence parametrized in $K$. It contains the key for answering the SK problem. Every deposit crystal A has the tendency to increase its height $h$ at constant number of atoms $N=h \ell^{2}$ since by this way it decreases the total strain energy. (In Fig. 2 refer to a curve $K$ and an original shape $h / \ell$ ). This is true provided the strain energy exists that means when there is a misfit $\mathrm{m}$ between the material $\mathrm{A}$ and the substrate 


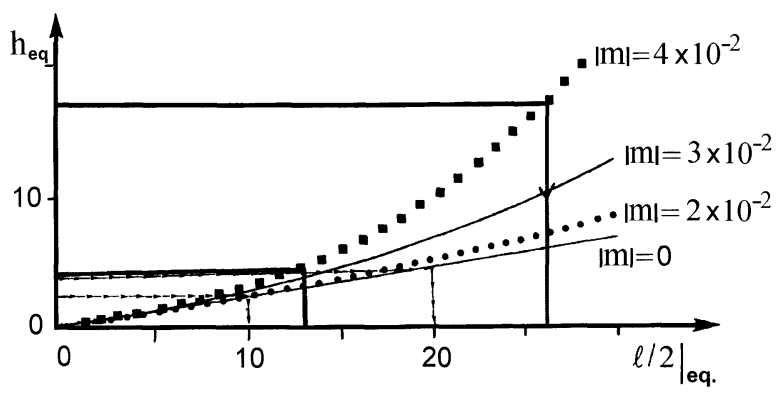

Fig. 3. - Equilibrium shape of a cubic crystal (height $h_{\mathrm{eq}}$, lateral half size $\ell_{\mathrm{eq}}$ ) for $K=1$ and different natural misfits $m$.

B. Therefore $W_{\min }$ at constant number of atoms in a $3 \mathrm{D}$ crystal is the thermodynamic driving energy for thickening. It opposes to the wetting energy which is still negative as we have shown at the beginning of this section. For definitely producing thickening there is also to overcome the lateral surface creation costs $\left(4 \sigma_{\mathrm{A}} \sqrt{N h}\right)$. The critical thickness of the SK transition therefore happens at some critical number of wetting layers $z_{\mathrm{SK}}$. This number can be calculated [6].

\section{Equilibrium Shape of a Crystal Deposited onto a Mismatched Substrate}

The equilibrium shape of a $3 \mathrm{D}$ crystal $\mathrm{A}$ in coherent epitaxy on its substrate $\mathrm{B}$ (or on its SK wetting layer coherently strained on B) can be calculated [16]. The principle is to write down all the free energy terms, including chemical potential $\Delta \mu$, for the crystal A containing $N=h \ell^{2}$ atoms and to vary separately $h$ and $\ell$. Combining both partial derivatives, there results the interesting relation [16] giving implicitely the equilibrium shape of the 3D crystal

$$
h_{\mathrm{eq}}=-\frac{4 \sigma_{\mathrm{A}}}{3 \varepsilon_{\mathrm{A}} m^{2} n_{\mathrm{s}}}\left(1-\frac{r_{0}}{r}\right) / \frac{\mathrm{d} R(r)}{\mathrm{d} r}
$$

where $r_{0}=h_{0} / \ell_{0}$, given by (2), is the shape ratio for non misfitted species $\mathrm{A}$ and $\mathrm{B}$ depending upon the wetting ratio $\left|\Phi_{\mathrm{AB}}\right| / 2 \sigma_{\mathrm{A}}$. On the contrary $r=h / \ell$ is the ratio when both species $\mathrm{A}$ and $\mathrm{B}$ have natural misfit $m . R(r)$ is the relaxation energy factor written in (5). This implicit relation can be solved and in Figure $3, h_{\mathrm{eq}}$ is plotted versus $\ell_{\mathrm{eq}} / 2$ so that half of the equilibrium shapes can easily be found as done for two sizes by heavy lines for $|m|=4 \times 10^{-2}$. Beneath the horizontal axis is the substrate $\mathrm{B}$. The curves are drawn parametrized by the misfit $|m|, K$ has been taken unity here. The curve $m=0$ is a straight line so that the equilibrium shapes have constant shape ratio $r_{0}=\left|\Phi_{\mathrm{AB}}\right| / 2 \sigma_{\mathrm{A}}$ we took equal to $1 / 10$. When the misfit is $m \neq 0$, what ever its sign, the shape is no more homothetic. For each misfit $m$ there is size dependent shape ratio, increasing with size. At a given lateral size, the shape ratio increases for increasing misfit $m$. Here is clearly illustrated the former sentence that strain opposes to wetting. Changing the relative stiffness $K$ entering in the $R$ factor, at a given misfit $m$ and wetting ratio $r_{0}$ or increasing the substrate stiffness increases the deviation from the $\left(m=0, r_{0}\right)$ shape.

Nevertheless, during crystal growth, the 3D islands, even when partially elastically relaxed accumulate a strain energy, which may become prohibitive. Therefore some plastic relaxation may occur and, in the case of interfacial dislocations, a similar treatment as in I has to be done. When an island grows on the nude substrate $\left(\Phi_{\mathrm{AB}}>0\right)$ dislocations introduce sideways 


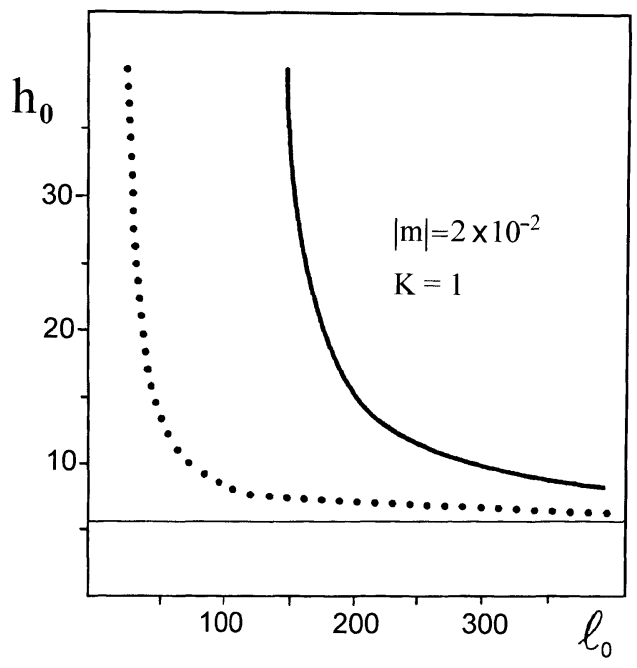

Fig. 4. - Critical height $h_{0}$ over critical lateral size dependence $\ell_{0}$ for the introduction of the first pair of dislocations. Full curve by considering elastic relaxation, doted curve without relaxation.

in the interface. Figure 1 has shown that at the borders of an island the lateral strain of the substrate B is highest and switches sign when passing the border $\left({ }^{4}\right)$. Just in the middle of the contact, lateral strain in the deposit $\mathrm{A}$ has its highest value so that the dislocation wanders inside releasing partially this strain. In the case of SK growth mode with $\Phi_{\mathrm{AB}}<0$ the situation is very similar, the dislocation wanders laterally in the interface of the island A and the wetting layer $\mathrm{A}$. When the wetting layers (at thickness $z_{\mathrm{SK}}$ but prior SK islands) form, their build up diminish the mean strain of the wetting layer $\left(^{5}\right)$ so that dislocations form in the A-B interface. The treatment is similar as in I, one finds:

$$
h_{N}=\frac{K}{4 \pi(1+K)} \frac{\ln \left(h_{N}+1\right)}{\left[m-(N+1 / 2) / \ell_{N}\right] R\left(h_{N} / \ell_{N}\right)}
$$

where $h_{N}$ and $\ell_{N}$ are the critical sizes (in atomic units) where the number $N$ of dislocations passed from $N$ to $N+1$. $N=0$ is the first introduction of a pair of orthogonal dislocations at size $h_{0}, \ell_{0} . R\left(h_{N} / \ell_{N}\right)$ is the energy relaxation factor of (5). In Figure 4 the critical values $h_{0}, \ell_{0}$ for the first introduction are given for a natural misfit $|m|=2 \times 10^{-2}$, we took also $K=1$. At the right there is the curve where the relaxation factor $R$ has been taken into account. The horizontal asymptot gives the critical thickness $h_{0} \approx 5$ for a continuous film $\ell_{0} \rightarrow \infty$ where also $R=1$. For islands of finite size $\ell_{0}$, greater critical thicknesses are allowed. These sizes increase rapidly for small islands close to $\ell_{0} \approx 150$. Smaller islands therefore resist much more to dislocation introduction compared to continuous layers. This is well confirmed by experiments. At the left of Figure 4 we drawn the $\left(h_{0}, \ell_{0}\right)$ dependence with the same parameters except we don't allowed the island to relax, maintaining $R=1$. This curve has, of course, the same horizontal asymptote than the former one, but it is shifted to the left up to a size $\ell_{0} \approx 30$. This is the result of Mathew's formulation [1] for islands where elastic relaxation is not considered.

$\left({ }^{4}\right)$ Shear strain in B close to the borders are also high with a maximum value a bit inside the bulk.

$\left({ }^{5}\right)$ At the contrary for the case $\Phi_{\mathrm{AB}}>0$ the island A creates strain in the substrate B initially strain free. 


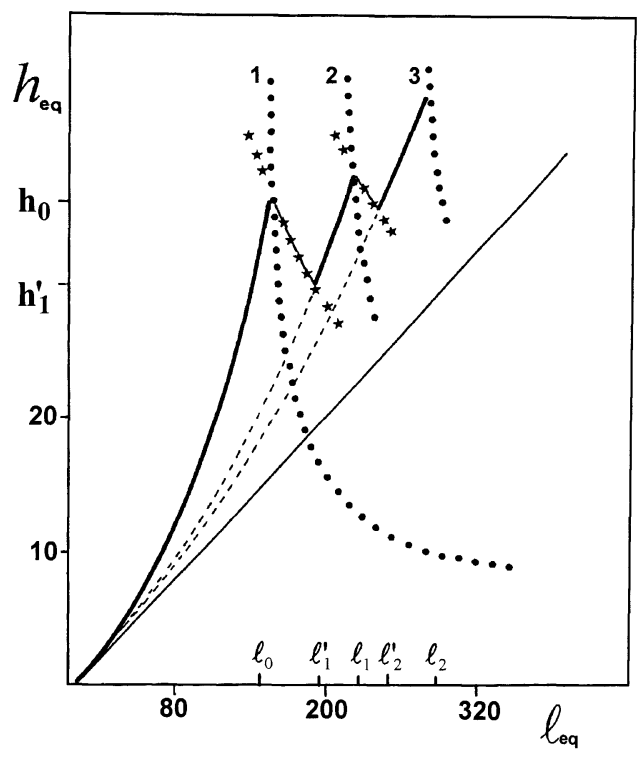

Fig. 5. - Equilibrium height $h_{\mathrm{eq}}$ versus lateral size $\ell_{\mathrm{eq}}$ of misfited $|m|=2 \times 10^{-2}$ coherent crystal on a substrate of relative stiffness $K=1$. The sawtooth behaviour of the shape, full curve, is due to the successive introduction, 1, 2, 3 of dislocations.

If one desires to give a scenario for introduction of successive dislocations during growth, one has to decide if this growth takes place either at constant height $h$ or at constant shape ratio $h / \ell$. This is quite a bit arbitrary but easily done by using graphs $h_{N}=f_{N}\left(\ell_{N}\right)$ from (6) similar to Figure 4. More meaningful is the case where growth takes place close to equilibrium, provided temperature is sufficiently high so that material transport by surface diffusion is efficient enough. This has been done in Figure 5 where $h$ and $\ell$ are the coordinates and $|m|=2 \times 10^{-2}, K=1$. From the left starts the equilibrium shape curve for a dislocation free crystal (heavy curve) which intersects the stability curve $N=0$ (that one of Fig. 4) coming from the right (dots) where the first dislocation may enter, let at size be $h_{0}, \ell_{0}$. Strain can release going from $m\left\langle M\left(h_{0} / \ell_{0}\right)\right\rangle$ to $\left(m-1 / \ell_{0}\right)\left\langle M\left(h_{0} / \ell_{0}\right)\right\rangle$ for the mean strain in the basal plane where $\langle M\rangle$ is the average value of $M(X)$ given by (4) for a length $\ell_{0}$. However now for this released strain the $h_{0} / \ell_{0}$ shape ratio is no more an equilibrium shape (since it is strain dependent) so that a new equilibrium shape curve, close to the former but just beneath (dashed), has to be drawn. How the 3D crystal goes from its former shape ratio to the new one is a matter of kinetics. When all changes happen quickly the island changes its shape practically at constant number of atoms there $h=h_{0} \ell_{0}^{2} / \ell_{1}^{2}=N / \ell^{2}$. The $1 / \ell^{2}$ decrease of the height is drawn (stars) to passing through $h_{0}, \ell_{0}$ and cuts the new equilibrium shape curve at $\left(\ell_{1}^{\prime}, h_{1}^{\prime}\right)$. In fact this drop of strain may be less rapid, however the new shape follows the second equilibrium shape curve. Successive introductions of dislocations have been constructed in Figure 5. The remarkable effect is that the equilibrium shape ratio of the crystal drops during each dislocation introduction and then increases again leading to a sawtooth behavior. This phenomenon has been experimentally observed very recently, in situ, on growing silicongermanium systems $[5,17]$. The associated strain-stress sawtooth oscillations can easily be drawn too. They have been observed since a long time [18]. Latter author was also the first who visualized by transmission electron microscopy the strain epitaxial islands generate in the substrate outside the contact areas. 


\section{Conclusion and Perspectives for non Isolated Crystals}

The elastic energy stored by coherent deposits on mismatched substrates leads to define several critical lengths. For $2 \mathrm{D}$ growth $\left(\Phi_{\mathrm{AB}}<0\right)$ we defined the specific number $z_{0}$ of wetting layers that exist at saturation, the critical number of layers $z_{\mathrm{dN}}$ at which interfacial dislocation may enter and that one at which a $3 \mathrm{D}$ growth is preferred $z_{\mathrm{SK}}$. For $3 \mathrm{D}$ growth on a nude substrate $\left(\Phi_{\mathrm{A}}>0\right)$ or onto $z_{\mathrm{SK}}$ sublayers $\left(\Phi_{\mathrm{A}}<0\right)$ the strain opposes to wetting and at some crystal size $(h, \ell)_{\mathrm{d}}$ the $3 \mathrm{D}$ crystal relaxes plastically by the introduction of dilocations.

Let us stress that the crystal relaxation leads to a substrate deformation beneath and outside the contact area. For example, strain and stress penetrate in the substrate up to $\ell / 2$ distances [16]. Then considering a collection of islands instead of an isolated one, there is an overlapping of their outside strain-stress fields $\varepsilon^{\mathrm{B}}$ as given by (4) and seen in Figure 1. But there is also superposition of strain field $\varepsilon^{\mathrm{A}}$ inside a given crystal with the outside strain field $\varepsilon^{\mathrm{B}}$ of the neighboring crystals.

In other words the islands communicate via the substrate. This overlapping induces some new interesting phenomena. For example if in an array of islands one of the island deviates in size or shape for some arbitrary reasons, its neighbors islands feel the change. Therefore install a driving force for material transport restoring a uniform size and shape distribution. At the same if the position of such an island deviates from its lattice site, the overlapping of the strain-stress field inside this crystal becomes asymmetric. Restoring forces ( gradient of chemical potential) drive the system to the minimal elastic and surface energy, that means to identical distance, size and shape. In other words the system by this communication avoids asymmetric situations coming back to the most symmetric situation. Then the elastic relaxation via the substrate deformation is the driving force for making auto-assembled crystal dots. The situation is quite different when dislocations introduce. Dislocations may install in such $3 \mathrm{D}$ crystals especially when the back-stress effect (communication) becomes very effective. Let us suppose that one of such a $3 \mathrm{D}$ crystal, in the regular array, releases its strain by two orthogonal dislocations. Its shape changes. It becomes flatter minimizing its elastic and its surface energy, atoms migrating from its top to its lateral faces. But it also received atoms from the immediate neighbor crystals since their free energy is higher. The array has a local irregularity: a much larger and flatter dislocated crystal (containing also a greater number of atoms) which is sitting between its neighbors (non dislocated crystals) containing fewer atoms than all the further-away neighbors of the array. This local situation is now highly irreversible. The dream of the high integrated circuit technology is it not to master such self assembled systems [11]?

\section{References}

[1] Mathews J.W., in "dislocations in solids", F. Nabarro Ed. (1979), p. 463 and in "Epitaxial growth", Vol A and B, Material Science Series (Acad. Press, 1975).

[2] van der Merve J.H. and Jesser W.A., J. Appl. Phys. 64 (1988) 4968.

[3] Kern R. et al., in "Current topics in material science", Vol.3, Kaldis Ed. (North Holland, 1979) p. 130.

[4] Kern R., in "Crystal growth in science and technology", Arend H. and Hulligen J. Eds. (Plenum Press, 1989) p. 143.

[5] Legoues F.K., MRS Bull (April 1996) 38. 
[6] Müller P. and Kern R., Appl. Surf. Sci. 102 (1996) 3; Kern R. and Müller P., Bulg. Chem Comm. 27 (1994) 24.

[7] Kern R. and Müller P., J. Cryst. Growth 146 (1995) 193.

[8] Kaishew R., Bull. Acad. Sci. Bulg. Serie Phys. 2 (1951) 191.

[9] Bauer E., Z. Krist 10 (1958) 372; Bauer E. et al., Surf. Sci. 53 (1975) 87.

[10] Ratsch C. and Zangwill A., Surf. Sci. 293 (1993) 123.

[11] Tersoff J. and Tromp R.M., Phys. Rev. Lett 70 (1993) 2782; Tersoff J. and Legoues F.K., Phys. Rev. Lett. 72 (1994) 3570.

[12] Duport Ch. et al., Phys. Rev. Lett. 74 (1995) 134.

[13] Hu S.M., J. Appl. Phys. 50 (1979) 4661.

[14] Kern R. and Müller P., Surf. Sci. 392 (1997) 103.

[15] Marchenko V.I., Sov. Phys. JETP 54 (1981) 761; JETP 33 (1981) 381.

[16] Müller P. and Kern R., J. Cryst. Growth (submitted).

[17] Hammar M., Legoues F.K., Tersoff J., Reuter M.C. and Tromp R.M., Surf. Sci. 349 (1996) 129.

[18] Vincent R., Phil. Mag. 19 (1969) 289.

[19] Niedermeyer R., Thin Sol. Films 1 (1968) 25. 